\title{
Evaluation of aggression and suicide attempt characteristics in patients with depression presenting with suicide attempt
}

\author{
Huseyin Murat Ozkan 1,*, Hamdullah Aydin ${ }^{2}$ \\ ${ }^{1}$ Istanbul Rumeli University, Faculty of Economics Administrative and Social Sciences, \\ Psychology Department, Silivri/Istanbul, Turkey \\ ${ }^{2}$ Altinbas University, Faculty of Economics, Administrative and Social Sciences, Psychology \\ Department, Istanbul, Turkey \\ *Corresponding author \\ Email: hmurat.ozkan@rumeli.edu.tr(Huseyin Murat Ozkan), hamdullahydn@gmail.com \\ (Hamdullah Aydin)
}

\begin{abstract}
OBJECTIVE: The assumption that aggression plays a role in suicidal behavior derives from the interpretations made due to low serotonin levels in suicide attempts and aggressive behaviors. In this study, it was aimed to examine the characteristics of aggression and suicide attempt in patients with depression presenting with suicide attempt.

METHODS: 101 depressive cases, who had suicide attempt and did not have any comorbid diagnosis, were included in the study while 54 depressive cases, who applied to psychiatry polyclinic, matched by age and gender, did not have suicide attempt and comorbid diagnosis, were included in the control group. Semi-structured history form, Hamilton Depression Scale and Ilter Kiper Aggression Scale were applied to the participants.
\end{abstract}


RESULTS: No difference was found between aggression subgroup scores and aggression total scores between the study group and control group. No difference was found between aggression and depression scores between the cases using aggressive and non-aggressive methods in suicide attempt. The disruptive aggression scores and aggression total scores of the cases with previous suicide attempt were found to be lower than the cases without previous suicide attempt. The assertiveness, passive aggression and aggression total scores of the cases consulting a doctor due to mental complaints before attempt were higher than those consulting with somatic complaints.

CONCLUSION: In studies, different psychiatric diagnoses and the relationship between aggression and suicide are emphasized. Aggression is among the basic motives of living beings and is a part of life. The inability to manage aggression rather than the presence of aggression seems to be a problem in suicidal behavior. Whether there is a unilateral or mutual relationship between aggression and psychiatric diagnosis and suicidal behavior is still a subject that needs further investigation.

Keywords: Suicide attempt, aggression, depression

\section{Introduction}

Suicide has been among the most important causes of death in human history $[1,2,3]$. Thus, in every period, suicidal behavior has been addressed with its social dimension as well as from the individual perspective. When the relationships of descriptive elements such as its frequency, form, factors that prepare and reveal it, comorbid diseases and socio-demographic characteristics are examined, it is understood that a factor is not explanatory alone $[4,5,6,7,8$, $9,10]$.

Although mental state and disorders can seem like the determinant of the behavior within the risk factors of suicide, social, biological, genetic and physical causes are also among the triggers of suicide. In mental disorders, suicidal behavior is mostly seen in depression [11, 12]. Depression has a separate place in psychiatric disorders due to its prevalence, genetic burden, the risk of recurrence, being an increasingly severe picture with each recurrence and having a more difficult treatment process. Aggression is among the basic motives of human beings. It is 
known to exist in the lives of humans in many stages such as protecting oneself, its destructiveness when reflecting on the outer world and injury/harm when directing to oneself as well as many other various forms. In this context, aggression is also addressed when defining mental disorders. The relationship between aggression and suicide in depression is included in theoretical definitions. The presence of aggression, implicitly or explicitly, at this point attracts attention in individual interviews. Studies examining the relationship between aggression and suicidal behavior suggest that depression cases with a history of suicidal ideation and behavior are more aggressive than those without suicidal ideation and behavior $[11,12,13,14]$. In these studies, although there is clearly hostility, it is pointed out that indirect aggression is more determinant in suicide attempt rather than the severity of depression $[15,16]$. Some of these studies investigating the relationship between aggression and suicide cover childhood and adolescence periods [17, 18, 19, 20, 21, 22]. In some studies, the relationship between aggression and suicide attempt is emphasized in cases with diseases with chronic pain, borderline personality as well as criminal and antisocial cases. Furthermore, the relationship between suicide and aggression has been reported in the studies conducted with healthy university students $[23,24,25]$.

In this study, it was aimed to examine the relationship between aggression and suicide attempt in depression patients, who attempted suicide, based on the sentence "aggression is directed to oneself in suicide".

\section{Methods}

This study was approved as a specialty thesis in medicine with the permission of the Gülhane Military Medical Academy (GATA)Department of Psychiatry (April 01, 1998, 0530-171-98). In this study, 101 consecutive patients diagnosed with depressive disorder among the patients who applied and/or were brought to Gülhane Military Medical Academy (GATA) Emergency Service between May 1999 and August 2001, and who were observed in the emergency department for at least 24 hours, were accepted as the study group. 54 depressive cases, who applied to psychiatry polyclinic, matched by age and gender and did not have suicide attempt, were included in the control group.

The patients, who were diagnosed with depression according to the DSM IV diagnosis criteria, treated and followed at least for 24 hours in intensive care units after suicide attempts, were 
included in the study group, while the depressive patients, who did not have suicide attempt, were included in the control group. The inclusion criteria were being between the ages of 1865 , receiving education at least for 5 years and being voluntary to participate in the study. Those, who were diagnosed with personality disorder and DSM IV Axis-I psychiatric diagnoses in addition to depression, who had a previous history of head trauma, who had nonsuicidal intoxication and who had mental retardation were excluded from the study.

The objective of the study was explained to all participants, and written informed consents were obtained from those who agreed to participate. Clinical interview was conducted, and SCID 1 was applied to all cases for diagnosis confirmation and exclusion. In the study group, psychiatric evaluations were conducted after their treatments in surgery clinics within two months after their attempt with firearm. In those, who had suicidal attempts with other methods, interviews were conducted 36-48 hours after the attempt after 24-hour short stay.

The cases, who attempted suicide, were questioned with the suicide history form to obtain information regarding sociodemographic characteristics and parameters related to the attempt. The Ilter Kiper Aggression Inventory, which is a self-reported scale, was applied to the cases to evaluate their aggression levels, and their depression levels were evaluated with Hamilton Depression Scale applied by the clinician.

\section{Assessment Tools}

Psychiatric Interview: Semi-structured clinical interview form (SCID-1), whose adaptation and reliability studies were conducted in our country, were applied to the cases following the formal psychiatric interview [26]. With SCID-1, all Axis 1 psychiatric pathologies are questioned within a modular structure in the last one month and throughout life.

Suicide History Form: This form was prepared by the researcher, and the parameters related to demographic information and suicidal behavior are evaluated. The form was applied by the researcher during the interview.

Hamilton Depression Rating Scale (HAM-D): It is a scale commonly used to detect the symptom severity in depressive patients. In this study, 17-item form of this scale was used. The form is filled out at the end of an unscheduled interview and aims to determine the severity of the patient's symptoms in the past few days [27]. 
Ilter-Kiper Aggression Inventory(I-KAI) [28]: For the questions related to disruptive aggression in this scale, 30 items were selected by benefitting from the aggression criteria found in "Personal Orientation Inventory", "Edwards Personal Preference Schedule" and "Kocatürk's Aggression Inventory". The first 10 items, which obtained the highest score in the field of assertive, passive and disruptive aggression, were selected, and a 30-question aggression inventory was developed. The questions were placed in consecutive order of threes, and the last three questions were placed one by one. The form has instructions at the beginning; it is an inventory that individuals will implement themselves. For each item, participants are asked to choose one of the seven options from "completely agree $(+3)$ " to "completely disagree $(-3)$. While rating, each sub-section is evaluated within themselves, and the scores to be used in statistical processes are obtained by adding 31 to the obtained score.

Test-retest reliability was performed by applying the inventory twice with 10 days intervals to the METU (Middle East Technical University) preparatory class students. The inventory's invariance coefficient calculated with the Pearson Product-Moment technique was detected as 0.81 in "disruptive aggression" subtest, 0.93 in "assertiveness" subtest and 0.88 in "passive aggression". For construct validity, the relationships among the three subtests were examined. The Pearson Product-Moment correlation coefficients of the inventory were -.30 between "disruptive aggression" and "assertiveness, -.38 between "disruptive aggression" and "passive aggression" and -.04 between "passive aggression" and "assertiveness" subtests.

Statistical Analysis: The data obtained were analyzed by using SPSS for Windows 10.0 package program. Chi-square, Independent samples t-test, One-Way ANOVA and correlation analysis were used for the statistical evaluation of the findings.

\section{Results}

The study group consisted of 101 cases as 65 males and 36 females, while the control group consisted of 54 cases as 36 males and 18 females. The mean age of the study group was $25.3 \pm$ 6.6 years, while the mean age of the control group was $27.16 \pm 5.6$ years (age range: 18-65 years).

No difference was found between aggression subgroup scores and aggression total scores between the study and control groups. No significant difference was determined between the 
study and control groups in terms of suicide attempt in family, family violence and family history of psychiatric treatment and difficult living conditions ( $p>.05)$.

The HAM-D total scores of the study group were higher than the control group $(\mathrm{p}<.01)$. (19.4 \pm 5.7 and $17.3 \pm 3.8$, respectively). HAM-D scores and Hamilton depression severity was evaluated by accepting the cut-off point as 16 points, and there was no significant difference between the study group and control group ( $p>.05)$.

When the suicide-related parameters were examined in the study group, the most frequently observed factor was drug-chemical substance use (74\%), while attempt with firearm (15\%) ranked number two. This was followed by attempt with sharp objects by $6 \%$, jumping from a high place by $3 \%$ and city electric current by $2 \% .31 \%$ of those, who attempted to suicide, left a suicide note. $34 \%$ of those, who attempted to suicide, had a previous suicide attempt. It was stated that $54 \%$ of those, who attempted to suicide, thought about the attempt, $41 \%$ suddenly decided and $5 \%$ planned the attempt. $29 \%$ of those, who attempted to suicide, stated that they told about these thoughts to the people around them before the attempt. While $50 \%$ of those, who attempted to suicide, stated that they had suicidal ideation in the last month, $14 \%$ stated that they had suicidal ideation within one to three months, and the remaining $36 \%$ for more than three months. When the suicidal ideation dimension was evaluated, $71 \%$ of those, who attempted to suicide, had short-term suicidal ideation, 22\% had long-term suicidal ideation, and $8 \%$ had persistent or almost continuous suicidal ideation. $52 \%$ of those, who attempted to suicide, consulted a doctor within the last month, 20\% within one-three months, and $29 \%$ within more than three months. $43 \%$ of the latest consultations were due to mental complaints. In the cases with previous suicide attempt, the number of the individuals consulting a doctor due to mental complaints was higher, while, in the cases without previous suicide attempt, the number of those with somatic complaints was higher ( $p<.05$, Chi-Square: 5.53$)$.

Comparison of aggression and depression scores by various parameters in the study group[Table 1]. 
Table 1: Comparison of aggression and depression scores by various parameters in the study group

\begin{tabular}{|c|c|c|c|c|c|}
\hline & $\begin{array}{l}\text { Disruptive } \\
\text { Aggression }\end{array}$ & Assertiveness & $\begin{array}{c}\text { Passive } \\
\text { Aggression }\end{array}$ & $\begin{array}{l}\text { Aggression } \\
\text { Total Scores }\end{array}$ & $\begin{array}{c}\text { HAM-D } \\
\text { Total Scores }\end{array}$ \\
\hline \multicolumn{6}{|c|}{ Difficult Living Condition: } \\
\hline Yes $\quad n=88$ & $35.1 \pm 11.6$ & $26.0 \pm 11.9$ & $23.9 \pm 11.2$ & $84.4 \pm 25.1$ & $19.3 \pm 5.6$ \\
\hline No $n=13$ & $31.2 \pm 6.1$ & $25.8 \pm 7.5$ & $25.3 \pm 9.8$ & $82.3 \pm 12.0$ & $19.5 \pm 6.5$ \\
\hline \multicolumn{6}{|l|}{ Aggressive Method } \\
\hline Yes $\quad n=26$ & $35.0 \pm 12.6$ & $25.6 \pm 11.4$ & $26.6 \pm 12.9$ & $86.8 \pm 28.5$ & $17.4 \pm 7.3$ \\
\hline No $\quad n=75$ & $34.5 \pm 10.6$ & $26.2 \pm 11.5$ & $23.1 \pm 10.2$ & $83.2 \pm 22.0$ & $20.0 \pm 5.0$ \\
\hline \multicolumn{6}{|l|}{ Suicide Note } \\
\hline Yes $n=31$ & $35.6 \pm 12.9$ & $27.5 \pm 11.8$ & $23.0 \pm 10.9$ & $85.5 \pm 23.9$ & $20.2 \pm 5.1$ \\
\hline No $\quad n=70$ & $33.7 \pm 11.4$ & $25.3 \pm 11.2$ & $24.5 \pm 10.1$ & $83.5 \pm 23.8$ & $19.0 \pm 5.9$ \\
\hline \multicolumn{6}{|l|}{ Previous attempt } \\
\hline Yes $\quad n=34$ & $29.4 \pm 12.1^{* *}$ & $23.6 \pm 11.8$ & $24.4 \pm 10.6$ & $76.6 \pm 23.1^{*}$ & $21.1 \pm 5.5^{*}$ \\
\hline No $\quad n=67$ & $37.2 \pm 9.7$ & $27.2 \pm 11.1$ & $23.8 \pm 11.2$ & $87.9 \pm 23.3$ & $18.5 \pm 5.6$ \\
\hline \multicolumn{6}{|c|}{ Impulsivity in Attempt: } \\
\hline Yes $n=41$ & $33.3 \pm 12.3$ & $26.9 \pm 13.2$ & $24.5 \pm 11.7$ & $84.6 \pm 26.6$ & $20.4 \pm 5.9^{*}$ \\
\hline No $n=60$ & $36.6 \pm 8.9$ & $24.7 \pm 8.1$ & $23.3 \pm 1010$ & $83.5 \pm 19.2$ & $17.9 \pm 5.2$ \\
\hline \multicolumn{6}{|c|}{ Mentioning about suicidal ideation: } \\
\hline Yes $n=29$ & $33.2 \pm 11.1^{*}$ & $25.3 \pm 11.4$ & $25.8 \pm 10.0$ & $84.1 \pm 21.8$ & $21.7 \pm 6.3^{*}$ \\
\hline No $\quad n=72$ & $35.1 \pm 11.2$ & $26.3 \pm 11.4$ & $23.3 \pm 11.3$ & $84.2 \pm 24.6$ & $18.4 \pm 5.2$ \\
\hline \multicolumn{6}{|c|}{ Suicidal ideation duration } \\
\hline In the last month & $35.7 \pm 10.6$ & $25.7 \pm 9.5$ & $23.2 \pm 10.9$ & $83.7 \pm 23.1$ & $17.4 \pm 4.4^{* *}$ \\
\hline More than a month & $33.5 \pm 11.6$ & $26.3 \pm 13.1$ & $24.9 \pm 11.1$ & $84.6 \pm 24.6$ & $21.4 \pm 621$ \\
\hline \multicolumn{6}{|c|}{ Reason for consulting a doctor } \\
\hline Mental $n=43$ & $32.5 \pm 12.3$ & $30.4 \pm 12.1 * *$ & $27.6 \pm 11.1^{* *}$ & $90.4 \pm 25.1^{* *}$ & $21.7 \pm 6.1^{* *}$ \\
\hline Somatic $\mathrm{n}=58$ & $36.2 \pm 10.0$ & $22.8 \pm 9.8$ & $21.4 \pm 10.3$ & $79.5 \pm 21.8$ & $17.6 \pm 4.7$ \\
\hline
\end{tabular}




\begin{tabular}{|c|c|c|c|c|c|c|}
\hline Yes & $\mathrm{n}=26$ & $27.1 \pm 11.8^{* *}$ & $28.1 \pm 12.8$ & $26.2 \pm 9.2$ & $81.4 \pm 20.8$ & $20.6 \pm 6.0$ \\
\hline No & $\mathrm{n}=75$ & $36.7 \pm 10.5$ & $25.2 \pm 10.9$ & $23.3 \pm 11.2$ & $85.1 \pm 24.7$ & $18.9 \pm 5.6$ \\
\hline \multicolumn{7}{|c|}{ Suicide Attempt in Family } \\
\hline Yes & $\mathrm{n}=18$ & $30.1 \pm 15.9$ & $24.1 \pm 10.1$ & $24.4 \pm 10.1$ & $80.6 \pm 23.4$ & $20.2 \pm 5.2$ \\
\hline No & $\mathrm{n}=83$ & $35.2 \pm 10.3$ & $26.4 \pm 11.0$ & $24.0 \pm 11.0$ & $85.0 \pm 24.0$ & $19.2 \pm 5.8$ \\
\hline \multicolumn{7}{|c|}{ Violence in Family } \\
\hline Yes & $\mathrm{n}=59$ & $34.4 \pm 12.5$ & $27.0 \pm 12.5$ & $25.6 \pm 11.5$ & $86.3 \pm 26.6$ & $18.6 \pm 4.7$ \\
\hline No & $\mathrm{n}=42$ & $34.1 \pm 10.4$ & $24.7 \pm 9.5$ & $21.8 \pm 10.0$ & $81.1 \pm 19.0$ & $20.4 \pm 6.9$ \\
\hline
\end{tabular}

$P>.05=$ Not significant $\quad P<.05, * *, \quad P<.01 \quad *$ : significant

When the difficult living conditions of the cases in the study group were evaluated, there was no difference between aggression and depression scores of the patients with and without difficult living conditions $(\mathrm{p}>.05)$.

When the methods used in the attempt were grouped as aggressive and non-aggressive methods, there was no difference between aggression and depression scores between the cases using aggressive and non-aggressive methods ( $p>.05)$.

No difference was found between the aggression and depression scores of those who left a suicide note and those who did not ( $p>.05)$. The mean disruptive aggression score and aggression total score of the study group cases with previous suicide attempt were found to be lower than the cases without previous suicide history $(p<.01)$. The Hamilton depression score and Hamilton depression severity of the cases with previous attempt were found to be higher compared to the cases without previous attempt $(\mathrm{p}<.01)$.

The mean Hamilton depression score of the study group cases stating that their attempts were sudden was higher than the cases with previous suicidal ideation $(p<.05)$. In the study group, the depression scores of the cases, who expressed their suicidal ideations to their environments before the attempt, were higher than those who did not $(p<.05)$, while the disruptive aggression scores of those who did not express their suicidal ideations were higher than those who did $(\mathrm{p}<.05)$.

In the study group, assertiveness $(p<.01)$, passive aggression $(p<.01)$, aggression total score ( $p$ $<.05)$ and Hamilton depression total score $(\mathrm{p}<.01)$ and Hamilton severity $(\mathrm{p}<.01)$ of the cases, 
who consulted a doctor due to mental complaints before the attempt, were higher than the patients consulting due to somatic complaints.

In the study group, disruptive aggression scores of those with mental treatment history in the family were lower than those who did not $(\mathrm{p}<.01)$. No difference was observed between other aggression scores and depression scores ( $p>.05)$. In the study group, no difference was found between the aggression and depression scores of the cases with and without violence history in family and suicide attempt history in family ( $\mathrm{p}>.05)$.

In the study group, a relationship was found between disruptive aggression and the total scores of assertiveness and aggression $(\mathrm{r}=.26, \mathrm{p}<.01$ and $\mathrm{r}=.62, \mathrm{p}<.01$, respectively). A relationship was found between disruptive aggression, passive aggression and aggression total scores $\mathrm{r}$ $=.26, \mathrm{r}=.39, \mathrm{r}=.78, \mathrm{p}<.01$, respectively). A relationship was found between passive aggression, assertiveness and aggression total scores $(\mathrm{r}=.39, \mathrm{p}<.01$ and $\mathrm{r}=.68, \mathrm{p}<.01$, respectively).

\section{Discussion}

The fact that the rate of the cases with previous suicide history in the study group is $34 \%$ and those who have attempted to suicide have a risk of retrying the attempt is consisted with the literature $[1,2,3]$.

No significant difference was observed between the study group and control group according to violence in family, attempt in family and psychiatric treatment in family. It can be stated that these factors do not explain the suicide attempt, point out pathologies in the family and set the ground for the emergence of depressive disorder, and suicidal behavior occurs when combined with other factors on this basis.

When the Hamilton depression scores of the control and study groups are examined, Hamilton depression scores of the cases, who attempted to suicide, are higher than others. The findings suggest that, although there is not a direct relationship with depression severity in suicide attempt, the risk is higher in severe depression.

When the total scores of disruptive aggression, assertiveness, passive aggression and aggression of the patients with and without suicide attempt were compared, a significant difference was not observed. This finding suggests that, although aggression generally does 
not seem to be a determining factor for suicide attempt in depressive patients, the aggression scale used in the study may be insufficient to measure aggression in this group.

When the sub-elements of aggression are examined in the study group, when the factors such as family structure and pathology in the family are considered, aggressive and disruptive aggression appears to be a factor leading to suicide in at least one group of patients. Based on the information in the literature, it can be stated that those, who attempted to suicide, are more aggressive, and their serotonin levels are low in depression and aggression [29, 30, 31, 32, 33]. On the other hand, we do not have enough information regarding the effect of aggression on psychiatric diagnoses and suicide attempts in healthy people. Some studies have examined aggression levels in healthy group [23, 24, 25].

When the methods used in the attempt are grouped as aggressive and non-aggressive methods, there is no difference between aggression and depression scores among the cases using aggressive and non-aggressive methods $(p>.05)$. In other words, aggression and depression scores do not indicate whether the chosen attempt method is aggressive or not. This suggests that the most important point is the suicide attempt, after the individual makes his decision, he chooses the easiest method; Therefore, a direct relationship with suicidal behavior cannot be established. From this perspective, it can be stated that, since they are among the easy-to-reach methods, drug-chemical substance use ranked first while firearm use ranked second because of working with soldier population.

$31 \%$ of those, who attempted to suicide, left a suicide note. No difference was observed between aggression and depression mean scores between the cases who left and those who did not leave a suicide note ( $p>.05$ ) [30]. Literature suggests that the content of the notes left by those committing suicide with aggressive methods is more aggressive than those not using aggressive methods [30]. $34 \%$ of those, who attempted to suicide, had a previous suicide attempt. Disruptive aggression scores $(p<.01)$, aggression total scores $(p<.05)$ of those with a previous suicide attempt were found to be lower, and their Hamilton depression total scores ( $p$ $<.05)$ and depression severities $(p<.05)$ were significantly higher compared to the group without a previous suicide attempt. Higher depression scores in those who had a previous attempt suggest that depression is deeper and longer. The low level of disruptive aggression in those with previous attempt may be related to the repetition of the attempt. In the literature, no information has been reached regarding the relation of previous attempt with aggression $[2,3]$. 
It was stated that $54 \%$ of those, who attempted to suicide, thought about the attempt, $41 \%$ suddenly decided and 5\% planned the attempt. The cases, who stated that their attempts were sudden, were evaluated as impulsive, while those, who planned to commit suicide, were evaluated as non-impulsive, and their aggression and depression scores were compared. No significant difference was observed between impulsive suicide attempt and aggression ( $p>.05)$. Depression scores of those evaluated as impulsive were higher than those who thought and planned the attempt $(\mathrm{p}<.05)$. This finding suggests that, as the severity of depression increases, the individual has difficulty in managing his reactions and aggression. In the study, no significant difference was observed between the cases with and without impulsive attempt in terms of assertiveness. The limited literature information on this subject indicates that depressive mood and impulsivity are associated with suicide attempt [29, 31, 32, 33]. The coexistence of aggression and suicide attempt seems to be more prominent in childhood and adolescence periods $[17,18,19,20,21,22]$.

In the study group, assertiveness $(\mathrm{p}<.01)$, passive aggression $(\mathrm{p}<.01)$, aggression total score ( $\mathrm{p}$ $<.05)$ and Hamilton depression total score $(p<.01)$ and Hamilton severity $(p<.01)$ of the cases with attempt, who consulted a doctor due to mental complaints before the attempt, were higher than the patients consulting due to somatic complaints. Consulting a doctor due to a mental complaint can be shown as an effort to look for help regardless of the dominance of disruptive aggression. High Hamilton scores indicate that this group suffers from a deeper depression and seeks help in touch with reality in a sense. Since the depressive cases in the control group are in the stage of somatizing their inner experiences, consulting a doctor seems to be far from being a mental-based step.

In those, who attempted to suicide, the rate of psychiatric treatment in the family is $25 \%$. When the cases, who had a history of psychiatric treatment in the family, were compared with those who did not have a history of psychiatric treatment in the family, the disruptive aggression scores of the former were found to be lower than the latter $(p<.01)$. This finding is significant when the relationship between suicide attempt and disruptive aggression is viewed from the perspective of psychiatric disorder in the family. In the cases without attempt, the difference emerges in terms of the passive aggression score in those who have a psychiatric disorder in the family $(\mathrm{p}<.01)$. In studies, presence of a psychiatric disorder in the family is seen as an essential factor in the context of depression and suicide attempt $[1,2,3]$. In addition to being an essential point in the depressive group, the point driving the individual to suicide 
shows itself in disruptive and passive sub-elements of aggression. In this study, depressive cases with and without suicide attempt were examined, and it can be stated that, as a result of the insufficient aggression management on a number of pathologies of attempt, disruptive aggression emerges and pulls the individual over death.

History of violence in the family was observed in $60 \%$ of those with suicide attempt. In the cases with suicide attempt, no difference was found between the aggression and depression scores of the cases with and without violence history in the family $(p>.05)$. History of suicide attempt in the family was observed in $18 \%$ of the cases who attempted to suicide. In the cases with suicide attempt, no difference was found between the aggression and depression scores of the cases with and without suicide history in the family ( $p>.05)$.

It is interesting that there is no difference between aggressive behaviors such as suicide attempt and violence in the family and the aggression scores of the cases included in the study. Classical information suggests that behavioral changes in the family may be related to aggression in those who attempt to suicide. While receiving psychiatric treatment in the family was found to be associated with aggression sub-scores, suicide attempt and violence in the family did not show significance. In the light of this information, it can be stated that psychiatric consultation has increased as an indicator of social development, pathology is distinct in cases with high need for seeking help, these pathologies may have been transmitted through genetics and identification, and this underlying experience leads to inadequacy in the management of aggression and suicide with the prominence of disruptive aggression.

A significant relationship was found between disruptive aggression and the total scores of assertiveness and aggression $(r=26, r=.62$, respectively, and $\mathrm{p}<.01$ in both). It can be said that the relationship between disruptive aggression and assertiveness and aggression total score shows that disruptive aggression is accompanied by assertiveness in the group with attempt, and perhaps, constitutes the main motive of the attempt. While there was no significant difference between the groups ( $p>.05)$, it can be stated that disruptive aggression and assertiveness act together and cause the individual to attempt to suicide. This attracts attention as a substantial finding that can shed light on understanding suicide.

A relationship was found between disruptive aggression and passive aggression $(r=.26, r=.39$, $\mathrm{p}<.01$ ). The relationship between assertiveness and passive aggression can be understood based on the fact that passive aggression and disruptive aggression describe an almost different 
way in the scale. The emergence of an activator element such as assertiveness together with disruptive and passive aggression explains the emergence of suicide attempt in depression. While a direction towards the action develops in one hand, disruptive and passive aggression determine the direction of the action on the other hand. In the depressed individual, the direction of the action is determined along the life-death line, and the action towards death seems to take place in the light of disruptive and passive aggression.

\section{Conclusion}

Aggression and depression scores do not determine the chosen attempt method. While it is seen that aggression does not have a role in impulsive attempt, factors such as violence in the family, suicide attempt in the family and psychiatric treatment history in the family do not explain aggression and suicide attempt, they indicate the pathologies in the family and/or pave the way for depression. When the total scores of disruptive aggression, assertiveness, passive aggression and aggression of the patients with and without suicide attempt were compared, no significant difference was observed. This finding suggests that, although aggression generally does not seem to be a determining factor for suicide attempt in depressive patients, the aggression scale used in the study may be insufficient to measure aggression in this group.

In the group with attempt, it can be stated that disruptive aggression and assertiveness act together and cause the individual to attempt to suicide. This attracts attention as a substantial finding that can shed light on understanding suicide.

Freud's approach suggests that aggression is directed inward in suicide. There are difficulties in defining and measuring aggression. Anger is aggression without an object. The observation of aggression as a part of life in healthy individuals suggests that the main problem is not the presence of aggression but the difficulty in managing aggression when psychiatric diseases and difficult life conditions are also considered.

Whether there is a unilateral or mutual relationship between aggression and psychiatric diagnosis, and suicidal behavior is still a subject that needs further investigation. 


\section{Highlights}

1-Aggression is among the basic motives of living beings and is a part of life.

2-The inability to manage aggression rather than the presence of aggression seems to be a problem in suicidal behavior

3- When the total scores of disruptive aggression, assertiveness, passive aggression and aggression of the patients with and without suicide attempt were compared, no significant difference was observed; suggesting that although aggression generally does not seem to be a determining factor for suicide attempt in depressive patients, the aggression scale used in the study may be insufficient to measure aggression in this group.

4- The cause and effect relationship between aggression and psychiatric diagnosis and suicidal behavior is still not adequately explained.

\section{References:}

[1] Say1l, I. (2000). Suicides. Mental Health and Disorders (p. 253-262). ANTIP A.Ş Publications Extended. 2nd Edition.

[2] Odağ, C. (1995). Suicide (Self-Destruction) Definition-Theory-Treatment. Psychiatric Association of Izmir, Department of Psychotherapies.

[3] Sadock, B. J., \& Sadock, V. A. (2000). Kaplan \&Sadock's Comprehensive Textbook Of Psychiatry (Vol I, p. 2031-4). Lippincot Williams\&Wilkins.

[4] Stone, M. (2016). Suicidality and aggression during antidepressant treatment: authors misinterpreted earlier paper from the FDA. BMJ, 206;352:i906.

[5] Tarang Sharma, L. S. (2016). Suicidality and aggression during antidepressant treatment: systematic review and meta-analyses based on clinical study reports. BMJ, 352-65.

[6] Amanda R. Start, Y. A. (2018). Predicting Suicide Ideation in the Military: The Independent Role of Aggression. Suicide and Life-Threatening Behavior. doi:10.1111/sltb. 12445

[7] Pilar Sierraa, L. L. ( 2011). Impulsivity, sensation seeking and aggressiveness in patients. Rev Psiquiatr Salud Ment (Barc.), 4(4):195---204. , Research and Practice, The British Psychological Society. doi:10.1111/papt.12123. 
[8] Gvion,Y., Horresh N, \& Levi-Belz, Y. (2014, Jan). Aggression-impulsivity, mental pain, and communication difficulties in medically serious and medically non serious suicide attempts. Compr Psychiatry(55(1)), 40-50. doi:10.1016/j.comppsych.2013.09.003

[9] Francesco Margari, M. L. (2014). Aggression, impulsivity, and suicide risk in benign chronic pain patients - a cross-sectional study. Neuropsychiatric Disease and Treatment, $101613-20$.

[10] Marc T. Swogger, Z. W. (2014 ). Reactive and proactive aggression and suicide attempts among criminal offenders. Crim Justice Behav., 41(3):337-344.

[11] Keilp, J., Gorlyn, M., \& Quendo, M. (2006, Dec). Aggressiveness, not impulsiveness or hostility, distinguishes suicide attempters with major depression. Psychol Med, 1779-88.

[12] Kang et al. (2015, Dec). Aggression, substance use disorder, and presence of prior suicide attempt among juvenile offenders with subclinical depression. (Kang et al. 2015) Law Hum Behav(39(6)), 593-601. doi:10.1037/lhb0000145. Epub 2015 Jul 13.

[13] Abay E, Tuğlu C. (2000). The Neurobiology of Violence and Aggression. J.Clin Psy., $3(1): 21-26$.

[14] Qquendo, M. A., \& Mann, J. J. (2000, Mar). The Biology Of Impulsivity And Suicidality. Clin North Am, 23(1), 11-25.

[15] Paul H. Soloff, L. C. (2010). Effects of serotonin-2A receptor binding and gender on personality traits and suicidal behavior in borderline personality disorder. j.pscychresns, 1234.

[16] Goldney, R., Winefield, A., Saebel, J., Winefield, H., \& Tiggeman, M. (1997, Sep-Oct). Anger, Suicidal Ideation And Attempted Suicide: A Prospective Study. Compr Psychiatry(38), 264-8.

[17] Castrogiovanni, P., Pieraccini, F., \& Di Muro, A. (1998, Feb). Suicidality And Aggressive Behavior. Acta Psychiatr Scand(97(2)), 144-8.

[18] Apter, A., Gothelf, D., Orbach, I., \& et al. (1995, Jul). Correlation of Suicidal And Violent Behavior in Different Diagnostic Categories in Hospitalized Adolescent Patients. J Am Acad Child Adolesv Psychiatry(34(7)), 912-8.

[19] Horesh, N., Gothelf, D., \& Ofek, a. a. (1999). Impulsivity as a Correlate of Suicidal Behavior in Adolescent Psychiatric Inpatients. Crisis(20), 8-14. 
[20] Kyung-Suk Lee, O.-J. C.-H. (2017). A Longitudinal Study on the Effects of Negative Rearing Experiences on Adolescents' Social Withdrawal and Aggression. Korean J Fam Med, 38:276-283.

[21] Girliani Silva de Sousa, M. S. (2017). Suicide in childhood: a literature review. Print version ISSN 1413-8123On-line version ISSN 1678-4561, 22(9):3099-3110. doi:10.1590/1413-81232017229.14582017

[22] Pratt, H. D., \& Greydanus, D. E. (2000, Feb). Adolescent Violence: Concept for a New Millennium. Adolesc Med, 11(1), 103-125.

[23] Seyed Ghafur Mousavi, K. K. (2011). Suicidal Ideation, Depression, and Aggression Among Students of Three Universities of Isfahan, Iran in 2008. Iran J Psychiatry Behav Sci, 6(1): 47-53.

[24] Wang L, He CZ, Yu YM et al. (2014). Associations between impulsivity, aggression, and suicide in Chinese college students. BMC Public Health. doi:https://doi.org/10.1186/1471-2458-14-551.

[25] Stanley, B., Molcho, A., Stanley, B., \& all. (2000, Apr). Association of Aggressive Behavior With Altered Serotonergic Function In Patients Who Are Not Suicidal. Am J Psychiatry, 157(4), 609-14.

[26] Çorapçıŏlu A, Aydemir Ö, \& Yıldız M. (1999). Structured clinical interview user guide for DSM-IV Axis I Disorders. Physicians Publication Association ("Hekimler Yayın Birliği")

[27] Önel, L. (1997). Psychological Tests Used in Turkey. Istanbul: Boğaziçi University Publications.

[28] Kiper, I. (1984). Examining the relationship of aggression types with various economic, social and academic variables. Unpublished master's thesis. Ankara University Institute of Social Sciences

[29] Carballo JJ, G.-N. R.-F., et al. (2014). Aggressiveness across development and suicidal behavior in depressed patients. Arch Suicide Res(18(1)), 39-49. doi:10.1080/13811118.2013.801808.

[30] Lester, D. (1998, Oct). The Content Of Suicide Notes Written By Using Different Methods. The Percept and Mot Skills, 87(2), 722. 
[31] Singh, P. K., \& Rao, V. R. (February 15 2018). Explaining suicide attempt with personality traits of aggression and impulsivity in a high risk tribal population of India. doi:https://doi.org/10.1371/journal.pone.0192969

[32] Iku Tsutsui-Kimura, Y. O. (2017). Milnacipran affects mouse impulsive, aggressive, and depressive-like behaviors in a distinct dose-dependent manner. Journal of Pharmacological Sciences, 134:181-189.

[33] Shri Cameron, V. J. (2017). Understanding the relationship between suicidality, current depressed mood, personality and cognitive factors. Psychology and Psychotherapy: Theory 